\title{
Session 7: Technology
}

[14.00-14.30]

'Monoclonal antibodies from plants - technical, regulatory and commercial feasibility'

Julian Ma

St George's University of London, London, UK

Abstract not provided.

[14.30-14.50]

'Next generation genome engineering of immune loci to generate therapeutics with novel properties' Lynn Macdonald

Regeneron Pharmaceuticals, Tarrytown, NY, USA

Abstract not provided.

[14.50-15.10]

'The bifunctional hybrid protein: A new enabling technology for non-linear epitope mapping'

Fabrizio Giannotta

ProGenosis SA, Liege, Belgium

Monoclonal antibodies (mAbs) are currently an area of intense research for human health. Determination of the region bound by the antibody paratope, i.e. the epitope, is pivotal for disease prevention, diagnosis and treatment, as well as for IP goal. Non-linear epitopes are an important form of epitope formed by residues that are sequentially discontinuous but close together in three-dimensional space. They have implicit structural information, making them attractive for both theoretical and applied biomedical research. These epitopes are hard to reconstruct using simple linear peptides, whose lack of structure prevents these peptides from being effective bio-molecules. The ProGenosis' Bifunctional Hybrid Protein (BHP) technology displays the secondary and tertiary structure of protein domains on the surface of a soluble and functional protein scaffold. These protein domains are constrained in a protein en- vironment. Consequently, the BHP epitope mapping approach is particularly suited to the determination of conformational epitopes.

In a recent PEDS publication, (In PEDS, 1-10, 2010), the BHP display methodology was used to elucidate a complex epitope on the extracellular domains of CD22, a specific marker on the surface of malignant B cells, in its interaction with the specific therapeutic antibody. The study showed that the interaction involves at least 3 discontinuous regions of the CD22 polypeptide and confirms the suitability of the BHP technology for rapid characterization of complex epitopes.

\section{[15.10-15.30]}

'New hybridoma technologies for producing monoclonal antibodies'

Masahiro Tomita and Kanta Tsumoto

Mie University, Tsu Mie, Japan

Monoclonal antibodies selectively recognizing specific epitopes in desired antigens have been employed for a multitude of purposes on the basis of specific interactions between antigens and antibodies. Already, several promising protocols have been shown capable of producing novel monoclonal antibodies. Among them, hybridoma technology is one of the most effective, utilizing innate functions of antibody-producing $B$ lymphocytes and cancerous cells to maximum advantage. Our group originally established an advanced hybridoma technology based on pre-selection of B lymphocytes with a target antigen and selective fusion of B cell-myeloma cell complexes by electrical pulses, allowing an at least ten times higher fusion efficiency than that obtained by the conventional approach. The advanced technology also facilitated selective and specific production of monoclonal antibodies against materials with low antigenicity.

Recently, we have succeeded in developing new protocols termed multi-targeting and stereo-specific targeting. Multi-targeting enables simultaneous produc- 
tion of monoclonal antibodies against multiple antigens. The point with this new technology is that single B lymphocytes theoretically produce single specific antibodies and each sensitized B lymphocyte harbors its own specific immunoglobulin receptors on its cell surface, so that co-culture of multiple cell lines allows mixtures of antibodies to be produced. The latter new advance features selective production of monoclonal antibodies specific to tertiary structures of target antigens. The critical point with this technology is the employment of antigen-expressing myeloma cells for selecting B lymphocytes. Since the antigens expressed on intact cells retain their original conformation, B lymphocytes selected by antigen-expressing myeloma cells based on immunoglobulin receptors may preferentially produce stereo-specific antibodies. This selective generation of conformation-specific monoclonal antibodies should find application for elucidating innate functions of antigens of interest, and also might have benefits for future medicines specifically targeting receptors on cancer cells.
[15.30-15.50]

'Probodies ${ }^{\mathrm{TM}}$ : A new generation of biologics engineered for tissue specificity'

Henry B. Lowman

CytomX Therapeutics Inc., South San Francisco, CA, USA

CytomX Therapeutics has devised a novel approach to developing protease-activated antibodies, termed Probodies $^{\mathrm{TM}}$. The antigen-combining site of an antibody is blocked with a masking peptide, which can be released by the action of endogenous proteases in diseased tissue. These molecules have the potential for reduced mechanism-based toxicities and increased therapeutic indices. This talk will describe our in vitro and in vivo approaches to characterizing Probody ${ }^{\mathrm{TM}}$ activity. 